Janette F. Walde

Design Künstlicher Neuronaler Netze 
WIRTSCHAFTSWISSENSCHAFT 


\author{
Janette F. Walde
}

\title{
Design Künstlicher Neuronaler Netze
}

Ein Leitfaden zur effizienten Handhabung mehrschichtiger Perzeptrone

Mit einem Geleitwort von Prof. Dr. Hans Werner Holub

Deutscher Universitäts-Verlag 
Bibliografische Information Der Deutschen Bibliothek

Die Deutsche Bibliothek verzeichnet diese Publikation in der Deutschen Nationalbibliografie; detaillierte bibliografische Daten sind im Internet über <http://dnb.ddb.de> abrufbar.

Dissertation Universität Innsbruck, 2000

\section{Auflage März 2005}

Alle Rechte vorbehalten

(C) Deutscher Universitäts-Verlag/GWV Fachverlage GmbH, Wiesbaden 2005

Lektorat: Ute Wrasmann / Britta Göhrisch-Radmacher

Der Deutsche Universitäts-Verlag ist ein Unternehmen von Springer Science+Business Media. www.duv.de

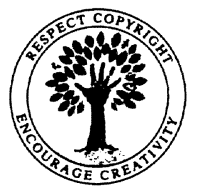

Das Werk einschließlich aller seiner Teile ist urheberrechtlich geschützt. Jede Verwertung außerhalb der engen Grenzen des Urheberrechtsgesetzes ist ohne Zustimmung des Verlags unzulässig und strafbar. Das gilt insbesondere für Vervielfältigungen, Übersetzungen, Mikroverfilmungen und die Einspeicherung und Verarbeitung in elektronischen Systemen.

Die Wiedergabe von Gebrauchsnamen, Handelsnamen, Warenbezeichnungen usw. in diesem Werk bërechtigt auch ohne besondere Kennzeichnung nicht zu der Annahme, dass solche Namen im Sinne der Warenzeichen- und Markenschutz-Gesetzgebung als frei zu betrachten wären und daher von jedermann benutzt werden dürften.

Umschlaggestaltung: Regine Zimmer, Dipl.-Designerin, Frankfurt/Main

Gedruckt auf säurefreiem und chlorfrei gebleichtem Papier

ISBN-13:978-3-8244-0842-9 e-ISBN-13:978-3-322-81211-7

DOI: $10.1007 / 978-3-322-81211-7$ 


\section{Geleitwort}

Künstliche Neuronale Netze (KNN) sind, wie so viele Methoden in der Ökonomik, eine Anleihe bei den Naturwissenschaften. Meiner Meinung nach handelt es sich dieses Mal aber um den „Import" einer außerordentlich interessanten Methode für die Ökonomen, vor allem für die Mustererkennung im weiteren Sinne. Dabei sind allerdings einige wichtige Anwendungsfragen noch ungeklärt, und wie bei allen neuen Instrumenten wird zuweilen auch Missbrauch damit getrieben.

Die Arbeit von Janette Walde setzt genau an diesen beiden Punkten an. Sie besteht inhaltlich aus zwei ineinander übergehenden Teilen:

- einmal einer übersichtlichen und kompetenten Darstellung bereits existierender Erkenntnisse zu Künstlichen Neuronalen Netzen,

- zum anderen der Entwicklung eigener weiterführender Forschungsergebnisse.

Beim ersten Punkt fällt vor allem die immense (man ist bei aller Vorsicht sogar geneigt zu sagen umfassende) Literaturkenntnis der Verfasserin auf. Dabei sind ersichtlich auch die Erkenntnisse mehrerer einschlägiger internationaler Konferenzen eingegangen.

Die wichtigen weiterführenden Forschungsergebnisse sind unter anderem:

- Überlegungen zur Mindestgröße der benötigten Stichproben,

- Überlegungen und Darstellung der Sättigungsmengen (mit besonderer Berücksichtigung ökonomischer Gegebenheiten),

- ausgiebige Vergleiche mit konventionellen linearen statistischen Verfahren (Diskriminanzanalyse, Regressionsanalyse etc.),

- Gegenüberstellung von Software - Paketen (SENN, Neural Connection, Neuframe) hinsichtlich Effizienz, Geschwindigkeit und Flexibilität,

- eine Analyse der Bedeutung gewählter Fehlerfunktionen,

- der Vergleich zweier Netzwerkarchitekturen für Klassifikationsfragen,

- der Nachweis von Instabilitäten der Performancekennzahlen, vor allem bei kleinen Stichprobengrößen, 
- die Konsequenzen der Stichprobengröße auf die Erreichbarkeit und Qualität der Ergebnisse und

- $\quad$ eine Diskussion der Problematik „verborgener“ (hidden) Neuronen.

Zusammenfassend kann man ohne Übertreibung feststellen, dass die vorliegende Arbeit mit Hilfe von analytischen und statistischen Methoden (anhand von realen und künstlichen Datensätzen) einige wichtige, bislang ungeklärte Fragen bezüglich KNN aufklärt und löst. Das wichtigste Ergebnis für Ökonomen ist sicherlich, dass (wie leider oft bei ökonomischen Anwendungen vorherrschend) kleine Datensätze nicht hinreichend sind, um die Vorzüge der nichtlinearen und nichtparametrischen $\mathrm{KNN}$ nutzen zu können. Oft sind hier sogar, wie die Verfasserin gezeigt hat, konventionelle statistische Verfahren überlegen.

Die Arbeit ist sehr gut lesbar und sie sollte zur Pflichtlektüre aller, an diesem neuen Instrument interessierten, Ökonomen gehören.

O. Univ.-Prof. Dr. Hans Werner Holub 


\title{
Vorwort
}

\begin{abstract}
„Annas Gesetz hieß: zuerst alles von innen nach außen, dann kopfüber-kopfunter, dann von hinten nach vorn, von rechts nach links. Und zum Schluss besah man sich das Ergebnis, und siehe da, es war ... “
\end{abstract}

Fynn, Hallo Mister Gott, hier spricht Anna

Diese Arbeit beschäftigt sich mit den Stärken und Schwächen von Künstlichen Neuronalen Netzen (KNN), insbesondere von den in der Praxis am häufigsten eingesetzten mehrschichtigen Perzeptronen, und versucht, die Fixierung der zahlreichen Freiheitsgrade (Anzahl der verborgenen Neuronen, Auswahl der Fehlerfunktion usw.) bei der Ausgestaltung der KNN von einer künstlerischen auf eine handwerkliche Basis zu stellen. Für dieses Unterfangen wird neben künstlich generierten Datensätzen auch ein realer Datensatz mit vollständigen Informationen zu mehr als 185.000 Beobachtungseinheiten verwendet. Dieser enorme Umfang sowie die Problemstellung der Klassifikation sind in idealer Weise dazu geeignet, die Chancen und Grenzen dieses Instrumentariums auszuloten. Neben dem Performancevergleich mit klassischen Verfahren werden mit Hilfe von Sensitivitätsanalysen die wichtigen Inputvariablen identifiziert und die aufgezeigten nichtlinearen Zusammenhänge auf ihre substanzwissenschaftliche Fundierung überprüft. Es werden Sättigungskurven erhoben und Sensitivitäten der Performancekennzahlen bezüglich Stichprobenauswahl im Vergleich zu klassischen Verfahren gemessen. Verschiedenste Einstellungen der Freiheitsgrade werden auf ihre Effizienz geprüft. Der eigentliche Vorzug der KNN - die bescheidene Vorabspezifizierung des funktionalen Zusammenhanges - geht, wie alle Ergebnisse dieser Arbeit zeigen, auf Kosten eines enormen Datenhungers, der in den meisten praktischen Anwendungen in der Ökonomie nicht gestillt werden kann.

Die vorliegende Arbeit wurde im Juli 2000 von der sozial- und wirtschaftswissenschaftlichen Fakultät der Leopold-Franzens-Universität Innsbruck als Dissertation angenommen. Sie 
entstand während meiner Tätigkeit als Universitätsassistentin am Institut für Statistik. Meinem wissenschaftlichen Lehrer, Herrn O. Univ.-Prof. Dr. Hans Werner Holub, danke ich für die Bereitstellung des Themas, seine Betreuung sowie sein reges Interesse am Fortgang der Arbeit. Herrn O. Univ.-Prof. Dr. Friedrich Roithmayr gebührt der Dank für die Übernahme des Zweitgutachtens. Für die zahlreichen Diskussionen und Anregungen sowie für die kritische Auseinandersetzung mit den Inhalten dieser Arbeit möchte ich ganz herzlich Herrn A. Univ.-Prof. Dr. Gottfried Tappeiner danken.

An dieser Stelle möchte ich mich auch bei Frau A. Univ.-Prof. Dr. Ulrike Tappeiner und Herrn Dr. Erich Tasser vom Institut für Botanik, Leopold-Franzens-Universität Innsbruck, bedanken für die Zurverfügungstellung des Datensatzes und für ihre intensive Zusammenarbeit. Für die Bereitstellung der Software SENN, mit der das Gros des Analyseteils berechnet wurde, möchte ich der Firma Siemens München, insbesondere Herrn Dr. Hans-Georg Zimmermann und Herrn Dr. Christoph Tietz, besonders danken.

Ein Dissertationsprojekt steht und fällt natürlich nicht nur mit dem wissenschaftlichen Umfeld, sondern mit den Menschen, die mich auf diesem Weg begleitet, unterstützt und immer wieder motiviert haben. Darum geht mein innigster Dank an meine Mutter (deren Glaube an mich eine wichtige Konstante in meinem Leben ist) und an meinen Ehemann Stefan (ohne den alles nichts wäre). 


\section{Inhaltsverzeichnis}

Inhaltsverzeichnis _ IX

Abbildungsverzeichnis__ XIII

Tabellenverzeichnis __ XV

Symbolverzeichnis__ XVII

Abkürzungsverzeichnis __ XIX

1 Einleitung _ 1

2 Das Mehrschichtige Perzeptron $(M L P) \_$_ 9

2.1 Dateninput _ 10

2.2 Verborgene Schicht

2.3 Outputschicht __ 15

2.4 Gewichte__ 18

2.4.1 Das Trainingsproblem 18

2.4.2 Die Validierungsmenge _ 22

2.4.3 Der Backpropagation Algorithmus _ 24

2.4.4 Der Lernalgorithmus _ 28

3 Standardprobleme beim Einsatz vom $M L P$

3.1 Aktivierungsfunktion 33

3.2 Selektion und Transformation von Inputvariablen __ 36

3.3 Anzahl der verborgenen Neuronen___ 38

3.4 Netzarchitektur __ 39

3.5 Fehlerfunktion

3.6 Stichprobengröße _ 46

3.7 Variabilität in den Performancekennzahlen __ 47 
$4 \quad K N N$-Software $\quad 49$

4.1 Software Vergleich bezüglich der Flexibilität __ 49

4.2 Leistungsfähigkeit der Programme __ 55

4.2.1 Höhe der Trefferquote __ 55

4.2.2 Schnelligkeit des Trainingsprozesses___ 56

5 Klassifikation mit Hilfe des Regressionsansatzes ___ 61

5.1 Datensatz 262

5.2 Festlegung des nichtlinearen Ansatzes ___ 66

5.2.1 Topologie und Netzspezifikationen __ 66

5.2.2 Multikollinearität___ 67

5.2.3 Lernstrategie _ 67

5.3 Leistungsfähigkeit 68

5.3.1 Trefferquote __ 68

5.3.2 Interpretation der Ergebnisse ___ 71

5.4 Einfluss der Stichprobengröße __ 76

5.4.1 Bestimmung der optimalen Anzahl verborgener Neuronen ___ 77

5.4.2 Sättigungskurve 80

5.5 Stabilität der Performancekennzahl ___ 82

5.5.1 Instabilität aufgrund der Gewichtsinitialisierung ___ 82

5.5.2 Instabilität aufgrund der Wahl der Stichprobe___ 83

5.6 Einfluss der Fehlerfunktion

6 Klassifikation mit Hilfe des wahrscheinlichkeitsbasierten Ansatzes___ 89

6.1 Festlegung des nichtlinearen Ansatzes ___ 89

6.2 Leistungsfähigkeit __ 91

6.2.1 Trefferquote ___ 91

6.2.2 Interpretation der Ergebnisse ___ 93

6.3 Einfluss der Stichprobengröße _ 100

6.3.1 Bestimmung der optimalen Anzahl verborgener Neuronen __ 100 
6.3.2 Sättigungskurve

6.4 Stabilität der Performancekennzahl 106

6.4.1 Instabilität aufgrund der Gewichtsinitialisierung 106

6.4.2 Instabilität aufgrund der Wahl der Stichprobe 107

6.5 Einfluss der Fehlerfunktion 109

6.6 Abschließender Vergleich der beiden $M L P$ Ansätze 110

7 Optimale Anzahl der verborgenen Neuronen 113

7.1 Hypothesen 114

7.2 Überprüfung mittels realem Datensatz 116

7.3 Künstliche Datensätze 118

7.3.1 Beschreibung der Datensätze 118

7.3.2 Ergebnisse 121

8 Schlussfolgerungen 125

Anhang A: Charakterisierung der Pflanzengesellschaften im Untersuchungsgebiet 133

Anhang B: Netzwerk Informationskriterium (NIC) 144

Literaturverzeichnis 145 


\section{Abbildungsverzeichnis}

Abbildung 1-1: Graphische Darstellung eines künstlichen Neurons. 2

Abbildung 1-2: Mögliche Darstellung der AND-Funktion mittels eines Neurons. ___ 2

Abbildung 1-3: Linear separables Problem und nicht linear separables Problem. ___ 3

Abbildung 1-4: Mehrschichtiges Neuronales Netz. ___ 4

Abbildung 1-5: Mögliche Darstellung der XOR-Funktion mit Hilfe eines Netzwerkes.__ 4

Abbildung 2-1: Vierschichtiges Perzeptron. ___ 9

Abbildung 2-2: Vollständige graphische Darstellung des Inputneurons $i$. 10

Abbildung 2-3: Tangens Hyperbolicus Funktion und Logistische Funktion. ___ 13

Abbildung 2-4: Vollständige mathematische Beschreibung des verborgenen Neurons $j$. _ 14

Abbildung 2-5: Netzwerk mit mehreren Inputclustern.

Abbildung 2-6: Elman Architektur und Jordan Architektur. ___ 16

Abbildung 2-7: Graphische Darstellung der Fehlerfunktion. ___ 19

Abbildung 2-8: Darstellung des Bias und der Varianz eines Modells. $\_22$

Abbildung 2-9: Kontrolle des Overfittings. ___ 23

Abbildung 2-10: Informationsfluss und Fehlerausbreitung in einem Netz. ___ 26

Abbildung 2-11: Konvergenzeigenschaften der drei vorgestellten Lernverfahren. ___ 29

Abbildung 3-1: Zweidimensionale achsensymmetrische Gaußfunktion. __ 34

Abbildung 3-2: Globale Struktur und Lokale Struktur. ___ 37

Abbildung 3-3: Netzwerk mit zusätzlicher linearer Struktur. ___ 41

Abbildung 5-1: Geographische Lage des Passeier Tals. ___ 63

Abbildung 5-2: Geographische Lage der Vegetationsklassen im Untersuchungsgebiet.__ 63

Abbildung 5-3: Vergleich der drei Fehlerfunktionen. ___ 71

Abbildung 5-4: Ziehen der Stichproben und Wahl der Generalisierungsmenge G2.___ 77

Abbildung 5-5: Sättigungskurven der beiden Verfahren (Regressionsansatz). ___ 82

Abbildung 5-6: Stabilität der Performancekennzahl in Abhängigkeit von verschiedenen Stichproben gleicher Größe für die Diskriminanzanalyse. ___ 84

Abbildung 5-7: Stabilität der Performancekennzahl in Abhängigkeit von verschiedenen

Stichproben gleicher Größe für das mehrschichtige Perzeptron. 85 
Abbildung 6-1: Sättigungskurven der beiden Verfahren (wahrscheinlichkeitsbasierter Ansatz). 104

Abbildung 6-2: Stabilität der Performancekennzahl in Abhängigkeit von verschiedenen Stichproben gleicher Größe für die Diskriminanzanalyse. 108

Abbildung 6-3: Stabilität der Performance in Abhängigkeit von verschiedenen Stichproben gleicher Größe für den wahrscheinlichkeitsbasierten Ansatz. 109

Abbildung 7-1: Abhängigkeit der Trefferquote von der Anzahl verborgener Neuronen. _ 113

Abbildung 7-2: Graphische Darstellung des ersten künstlich generierten Datensatzes. _ 119

Abbildung 7-3: Graphische Darstellung des zweiten künstlich generierten Datensatzes. _ 120

Abbildung 7-4: Graphische Darstellung des dritten künstlich generierten Datensatzes. 120

Abbildung 7-5: Darstellung des positiven Zusammenhangs zwischen der optimalen Zahl an verborgenen Neuronen und der erkannten Nichtlinearität. 


\section{Tabellenverzeichnis}

Tabelle 4-1: Vergleich der Flexibiltät von SENN, Neural Cornection und Neuframe.__ 54

Tabelle 4-2: Auflistung der Anwendungsbeispiele für den Softwarevergleich. ___ 57

Tabelle 4-3: Leistungsvergleich der drei Softwareprodukte für $n=1.000$. 57

Tabelle 4-4: Leistungsvergleich der drei Softwareprodukte für $n=100$. _ 59

Tabelle 4-5: Leistungsvergleich der drei Softwareprodukte für $n=10.000$. 60

Tabelle 5-1: Häufigkeitsverteilung der Vegetationsklassen.____ 64

Tabelle 5-2: Einflussfaktoren auf die Vegetationsmuster. ___ 65

Tabelle 5-3: Multikollinearitätsdiagnose der unabhängigen Variablen. ____ 67

Tabelle 5-4: Vergleich der Trefferquote der Diskriminanzanalyse und des MLPs. __ 72

Tabelle 5-5: Zentroidenvergleich für die Klassen 5 und 11 . ___ 74

Tabelle 5-6: Zentroidenvergleich für die Klassen 9 und 11 . _ 75

Tabelle 5-7: Zentroidenvergleich für die Klassen 10 und 11 . 75

Tabelle 5-8: Trefferquote bei $n=100$ abhängig von der Zahl verborgener Neuronen.__ 78

Tabelle 5-9: Trefferquote bei $n=250$ abhängig von der Zahl verborgener Neuronen.__ 78

Tabelle 5-10: Trefferquote bei $n=500$ abhängig von der Zahl verborgener Neuronen.___ 79

Tabelle 5-11: Trefferquote bei $n=1.000$ abhängig von der Zahl verborgener Neuronen. _ 79

Tabelle 5-12: Trefferquote bei $n=1.500$ abhängig von der Zahl verborgener Neuronen. _ 80

Tabelle 5-13: Instabilität der Trefferquote aufgrund der Gewichtsinitialisierungen. ___ 83

Tabelle 5-14: Ab welcher Stichprobengröße übertraf das Netz signifikant die Trefferquote der Diskriminanzanalyse? 86

Tabelle 5-15: Abhängigkeit der Trefferquote von der gewählten Fehlerfunktion. ___ 87

Tabelle 6-1: Verteilung der nicht eindeutig zugeordneten Beobachtungseinheiten. ___ 91

Tabelle 6-2: Zentroiden/Medoidenvergleich der nicht eindeutig zugeordneten Einheiten._ 92

Tabelle 6-3: Vergleich der Trefferquote der Diskriminanzanalyse und des MLPs (wahrscheinlichkeitsbasierter Ansatz) für jede Klasse.___ 94

Tabelle 6-4: Zentroidenvergleich für die Klassen 5 und 11 . ___ 97

Tabelle 6-5: Zentroidenvergleich für die Klassen 9 und 11 .___ 98

Tabelle 6-6: Zentroidenvergleich für die Klassen 10 und 11 .____ 98

Tabelle 6-7: Trefferquote bei $n=100$ abhängig von der Zahl verborgener Neuronen.__ 101 
Tabelle 6-8: Trefferquote bei $\mathrm{n}=175$ abhängig von der Zahl verborgener Neuronen. 101

Tabelle 6-9: Trefferquote bei $n=250$ abhängig von der Zahl verborgener Neuronen. 102

Tabelle 6-10: Trefferquote bei $n=500$ abhängig von der Zahl verborgener Neuronen. 102

Tabelle 6-11: Trefferquote bei $n=1.000$ abhängig von der Zahl verborgener Neuronen. _ 103

Tabelle 6-12: Trefferquote bei $n=1.500$ abhängig von der Zahl verborgener Neuronen. _ 103

Tabelle 6-13: Instabilität der Trefferquote aufgrund der Gewichtsinitialisierungen. 107

Tabelle 6-14: Abhängigkeit der Trefferquote von der gewählten Fehlerfunktion. 110

Tabelle 6-15: Abschließender Vergleich der beiden Netzwerkarchitekturen. 111

Tabelle 7-1: Abhängigkeit des Anteils der erkannten (Nicht)Linearität von der optimalen Anzahl von verborgenen Neuronen bei $n=250$. 116

Tabelle 7-2: Abhängigkeit der erkannten Nichtlinearität von der Stichprobengröße und der optimalen Zahl an verborgenen Neuronen.

Tabelle 7-3: Trefferquote der Diskriminanzanalyse für jede Stichprobengröße und für alle drei künstlich generierten Datensätze.

Tabelle 7-4: Abhängigkeit der optimalen Zahl verborgener Neuronen von der Stichprobengröße und dem Grad der Nichtlinearität für den ersten Datensatz. 121

Tabelle 7-5: Abhängigkeit der optimalen Zahl verborgener Neuronen von der Stichprobengröße und dem Grad der Nichtlinearität für den zweiten Datensatz. _ 122

Tabelle 7-6: Abhängigkeit der optimalen Zahl verborgener Neuronen von der Stichprobengröße und dem Grad der Nichtlinearität für den dritten Datensatz.

Tabelle 7-7: Abhängigkeit der optimalen Zahl verborgener Neuronen vom Grad der abgebildeten Nichtlinearität. 123 


\section{Symbolverzeichnis}

Für die gesamte Arbeit gilt, dass fett gedruckte Kleinbuchstaben Vektoren und fett gedruckte Großbuchstaben Matrizen kennzeichnen.

$\boldsymbol{w} \quad$ Parameter/Gewichtsvektor

$\boldsymbol{x} \quad$ Inputvektor/Eingangssignale

$y \quad$ Abhängige Variable

$\hat{y} \quad$ Geschätzter Wert für die abhängige Variable

$f(\boldsymbol{x}, \hat{\boldsymbol{w}}) \quad$ Netzoutput

$\theta \quad$ Schwellenwert

I Anzahl der Inputneuronen

$H \quad$ Anzahl der verbogenen Neuronen

$O \quad$ Anzahl der Outputneuronen

$\varepsilon \quad$ Stochastischer Fehlerterm, Rauschen

$P \quad$ Anzahl der Netzwerkparameter

$\mathfrak{R} \quad$ Menge der reellen Zahlen

$T \quad$ Menge der Trainingsdaten

$|T| \quad$ Zahl der für das Training zur Verfügung stehenden Trainingsdaten

$V \quad$ Validierungsmenge

$G \quad$ Generalisierungsmenge

$E(\boldsymbol{w}) \quad$ Fehlerfunktion in Abhängigkeit der Parameter $\boldsymbol{w}$

$\boldsymbol{a}^{T} \quad$ Transponierter Vektor $\boldsymbol{a}$

$|a| \quad$ Absolutbetrag des Skalars $a$

[a] Größtes Ganzes des Skalars $a$

$\varnothing \quad$ Durchschnitt

p Prozentsatz

$r \quad$ Korrelationskoeffizient

$r^{2} \quad$ Bestimmtheitsmaß/Determinationskoeffizient

$n \quad$ Stichprobengröße

$\bar{x} \quad$ Stichprobenmittelwert

$\hat{\sigma}_{\bar{x}} \quad$ Geschätzter Standardfehler der Mittelwerteverteilung

$\wedge \quad$ Logisches UND

$\checkmark \quad$ Logisches ODER 


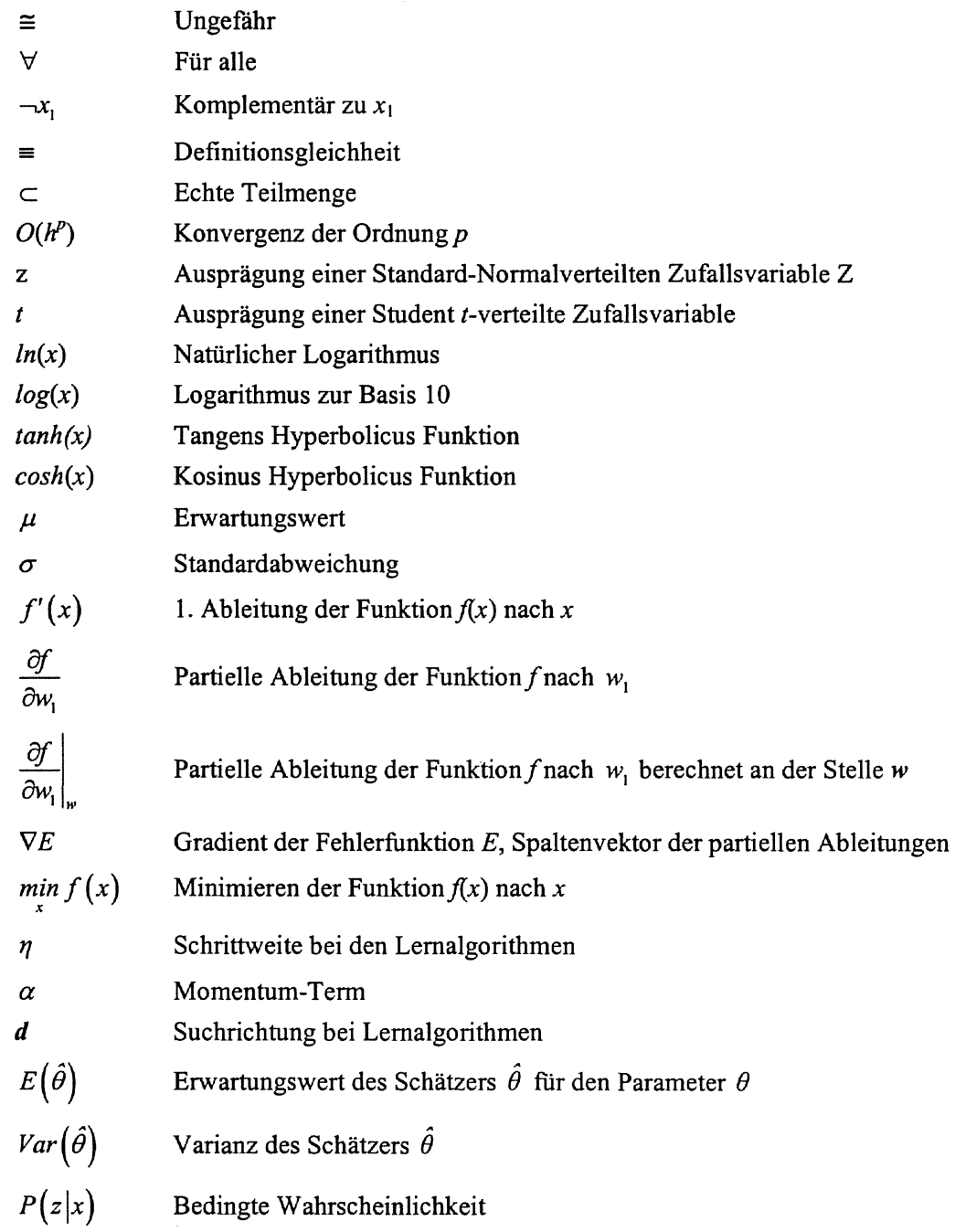




\section{Abkürzungsverzeichnis}

$\begin{array}{ll}A I C & \text { Akaike Informationskriterium } \\ A N D & \text { Boolsche } A N D \text {-Funktion } \\ A R I M A & \text { Autoregressive, integrierte, moving average Modelle } \\ B F G S & \text { Quasi-Newton-Methode nach Broyden, Fletcher, Goldfarb und Shanno } \\ B P & \text { Backpropagation Algorithmus } \\ C P U & \text { Central processing unit } \\ D A & \text { Diskriminanzanalyse } \\ G I S & \text { Geographisches Informationssystem } \\ H F & \text { Heaviside-Funktion } \\ K M C & \text { K-Means-Clustering Algorithmus } \\ K N N & \text { Künstliche Neuronale Netze } \\ L & \text { Likelihoodfunktion } \\ M L P & \text { Mehrschichtiges Perzeptron } \\ M S E & \text { Mean Squared Error, mittlerer quadratischer Fehler } \\ N C & \text { Neural Connection } \\ N I C & \text { Netzwerk Informationskriterium } \\ N F & \text { Neuframe } \\ P S C & \text { Predictive Stochastic Complexity Kriterium } \\ R B F & \text { Radiale Basisfunktionen } \\ R M S E & \text { Wurzel aus dem } M S E \\ S I C & \text { Schwarz-Informationskriterium } \\ X O R & \text { Boolsche } X O R \text {-Funktion }\end{array}$

